\title{
An unusual case of unilateral limb hypertrophy: Lipoma of sacral roots
}

\author{
Archana B Netto, Sanjib Sinha, Arun B Taly, Chandrajit Prasad'ㄹ, A Mahadevan², PS Bindu, Ravinder J Sidhu, \\ TC Yasha ${ }^{2}$ \\ Departments of Neurology, ${ }^{1}$ Neuroimaging and Interventional Neuroradiology, and ${ }^{2}$ Neuropathology, National Institute of Mental Health and \\ NeuroSciences (NIMHANS), Bangalore, India
}

\section{ABSTRACT}

We report an unusual case of unilateral limb pseudo hypertrophy in a 21-year-old lady who developed progressive enlargement of the right calf followed by thigh in association with chronic leg pain. Magnetic resonance imaging (MRI) of the affected limb confirmed enlargement of various muscles. Electromyography revealed neurogenic features consistent with Si radiculopathy. MRI of the lumbosacral spine showed tethered cord with a lipoma infiltrating multiple sacral roots. Our case illustrates that muscular pseudo hypertrophy may follow chronic denervation as a consequence of spinal neural compressive disease. The various mechanisms postulated for this distinct condition are outlined.

Key words: Cord lipoma, limb hypertrophy, neurogenic, tethered cord

\section{Introduction}

Muscle hypertrophy is the normal physiological response to work and results from the enlargement of individual myofibers. It has also been described in both myopathic and neurogenic disorders. The myopathic disorders in which it is seen include myotonia congenita, muscular dystrophy, hypothyroidism, acromegaly, inflammatory myopathies, Pompe's disease and proximal myotonic myopathy among others. The occurrence of muscle hypertrophy with neurogenic disorders (i.e., involving motor neurons or their axons) is distinctly unusual, muscle atrophy being the rule. However, muscle hypertrophy has been occasionally associated with neuropathies, radiculopathies, spinal muscular atrophy, and post-polio syndrome. ${ }^{[1]}$

This report highlights association of unilateral limb pseudo hypertrophy in patient with sacral root lipoma.

\begin{tabular}{|l|l|}
\hline \multicolumn{2}{|c|}{ Access this article online } \\
\hline Quick Response Code: & Website: \\
\hline & www.ruralneuropractice.com \\
\cline { 2 - 2 } & \\
\hline & \\
\hline
\end{tabular}

\section{Case Report}

A 21-year-old lady was referred to us for management of pain and paraesthesia involving the outer aspect of right ankle joint of approximately 18 months duration. Over the next few months she began to walk with a slight limp and the paraesthesia had extended to involve the whole of outer aspect of right leg. She consulted an orthopedician, who, noting nothing significantly wrong with her skeletal system prescribed analgesics and crepe bandage. Over the next few weeks she had difficulty in standing on toes while stacking some shelves at her house. Since the last 6 months she noticed that her pyjamas were becoming tighter at the right calf and within few weeks it started becoming tight at the level of thigh as well. Being medical personnel herself, she measured width of both calves and found the right calf to be $3.5 \mathrm{~cm}$ wider than the left. A magnetic resonance imaging (MRI) scan of the lower limbs was carried out at another hospital and was referred to us following muscle biopsy of the right calf. There was no other significant history.

She was moderately nourished lady with an antalgic gait, favoring the right leg. Motor examination revealed pseudo hypertrophy of right gastrocnemius and hamstring group of muscles with weakness of plantar flexion causing inability to walk on toes. Ankle dorsiflexor

\section{Address for correspondence:}

Dr. Sanjib Sinha, Department of Neurology, National Institute of Mental Health and Neurosciences (NIMHANS), Hosur Road, Bangalore - 560 029, India. E-mail: sanjib_sinha2004@yahoo.co.in 
strength was within normal limits as was testing of the proximal lower extremity muscle groups. The Achilles reflex was not obtainable on the right side even with the Jendrassik maneuver. The left Achilles reflex was normal as were other stretch reflexes. The sensory examination revealed a deficit to pin prick in the S1 dermatomal distribution on the right and intact vibratory and position sense. The straight leg-raising test was negative on both sides. There were no neurocutaneous markers noted and the systemic examination was unremarkable. In summary, the patient had manifested with unilateral S1 radiculopathy.

Nerve conduction studies [Table 1] showed low amplitude of compound muscle action potential in right posterior tibial nerve $(1.3 \mathrm{mV})$ as compared to left posterior tibial nerve $(14 \mathrm{mV})$ with normal sensory conductions (bilateral sural and superficial peroneal) suggesting an axonopathy involving the S1 and S2 roots. Needle electromyography revealed denervation of the right gastrocnemius. MRI (lumbosacral spine; Figure $1 \mathrm{~A}$ and $\mathrm{B}$ ) revealed a very well circumscribed intradural extramedullary $\mathrm{T} 1$ hyperintense [Figure 1A] and T2 isointense [Figure 1B] lesion at the level of body of S1 and S2 with scalloping of the posterior border of these two vertebrae. The lesion appeared to be arising from the sacral roots. The cord was tethered, and ended at S2. There was no enhancement on postcontrast fat saturated T1W images [Figure 1D]. The imaging features were suggestive of lipoma. MRI of both the calves revealed enlargement of gastrocnemius and thigh muscles with fatty replacement of muscle tissue [Figure 1E].

Biopsy of the enlarged right gastrocnemius muscle revealed infiltration of adipose tissue splaying apart the myofibres [Figure 2b]. There was evidence of a neurogenic process with denervation causing atrophy and myonuclear clumps. In addition, myopathic features with scattered fibers showing myophagocytosis and regeneration [Figure 2a] and focal interstitial inflammation was present close to aponeurosis with histiocytes and eosinophils. Features were consistent with focal myositis with denervation.

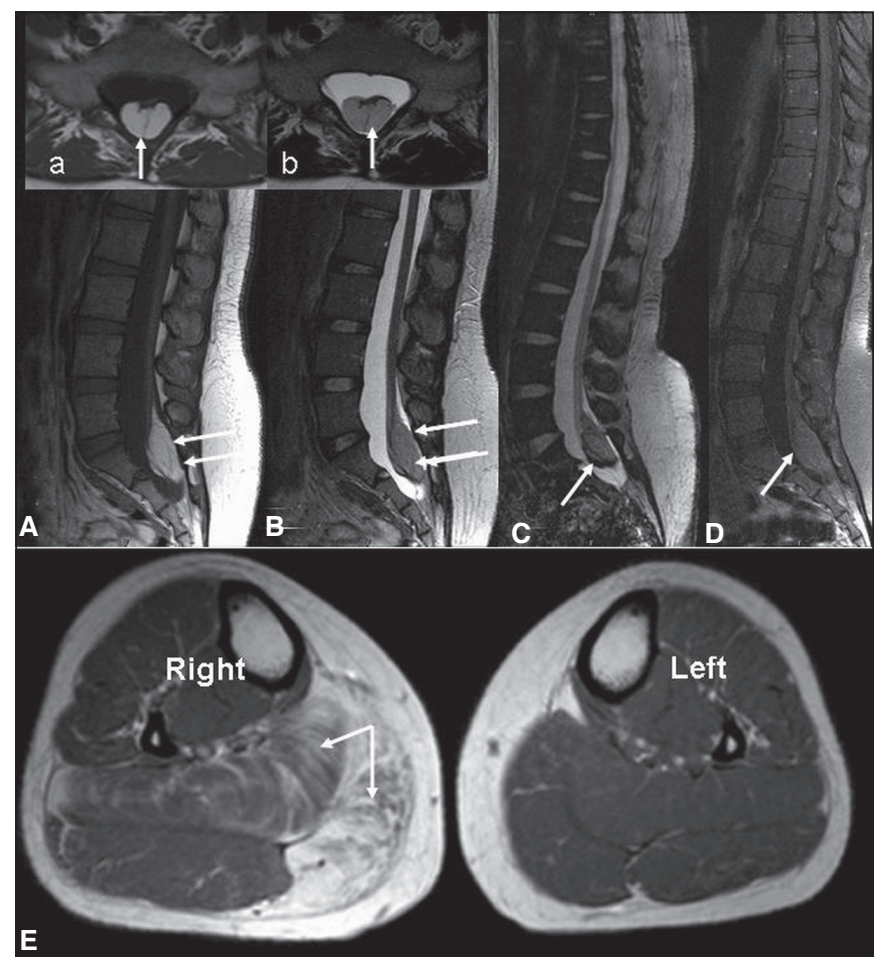

Figure 1: MRI of lumbosacral spine (1A-D) revealed a very well circumscribed intradural extramedullary $\mathrm{T} 1$ hyperintense $(\mathrm{A}, \mathrm{a}), \mathrm{T} 2$ isointense $(1 \mathrm{~B}, \mathrm{~b})$ lesion at the level of $\mathrm{S} 1$ and $\mathrm{S} 2$ with scalloping of the posterior border of these two vertebrae; along with chemical shift artefact on gradient imaging (1C); There was no enhancement on post-contrast fat saturated T1W images (1D). MRI of both the calves (1E) revealed enlargement of right calf with involvement of soleus and medial head of gastrocnemius with fatty replacement of muscle tissue.

Table 1: Nerve conduction study of the lower limbs

\begin{tabular}{lcccc}
\hline Nerves studied motor & Latency $(\mathbf{m s})$ & Amplitude $(\mathbf{m V})$ & Distance $(\mathbf{m m})$ & Nerve conduction velocity $(\mathbf{m} / \mathbf{s})$ \\
\hline Right CP & 3.8 & 11.2 & 90 & 48.1 \\
Right PT & 9.2 & 10.0 & 260 & 46.9 \\
& 2.1 & 1.3 & 85 & 50.0 \\
Left CP & 10.2 & 1.0 & 380 & 40 \\
& 4.2 & 7.7 & 265 & 49.4 \\
Left PT & 9.5 & 7.2 & 90 & 590 \\
Sensory & 4.1 & 17.5 & & 59.5 \\
Right superficial peroneal & 12.0 & 16.1 & 110 & 59.1 \\
Left superficial peroneal & 1.85 & 35 & 130 & 56.0 \\
Right sural & 2.2 & 32 & 140 & 45.5 \\
Left sural & 2.5 & 34 & 100 & \\
\hline
\end{tabular}

CP: common peroneal nerve, $\mathrm{ms}$ : milliseconds, $\mathrm{mm}$ : millimetres, $\mathrm{m} / \mathrm{s}$ : meter per second, $\mathrm{mV}$ : millivolts, PT: posterior tibial nerve 


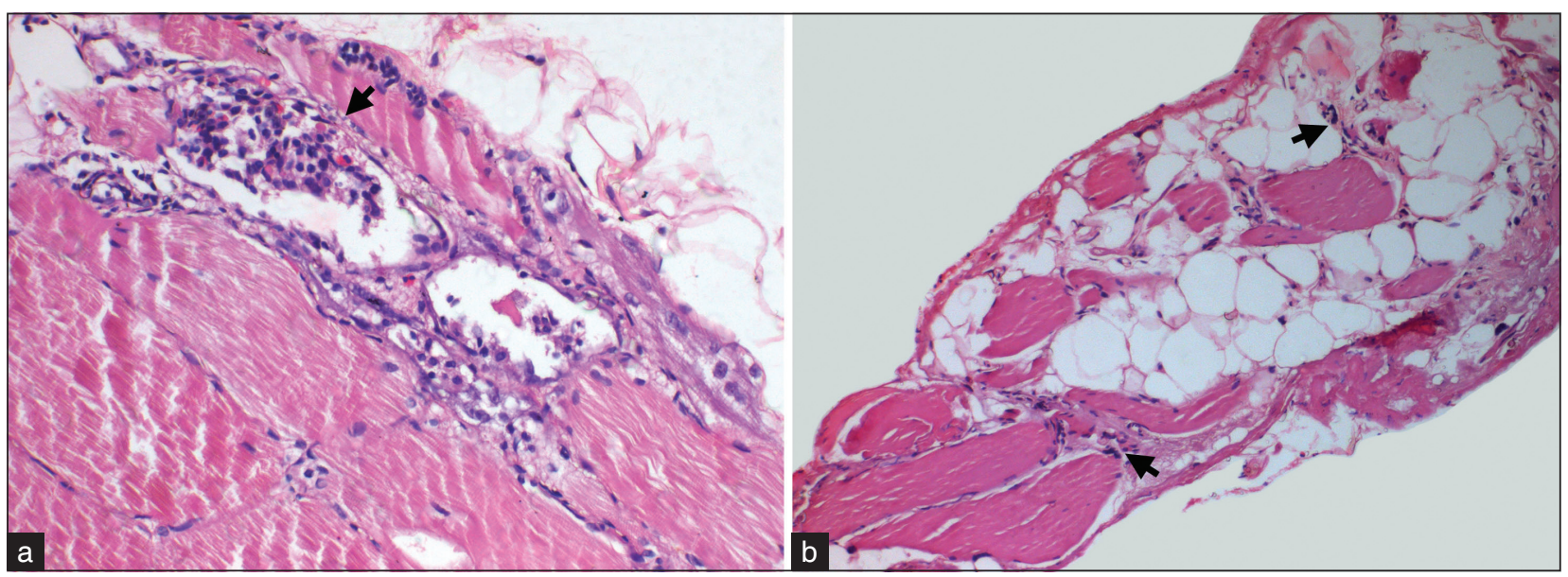

Figure 2: Muscle biopsy from right gastrocnemius revealed myopathic features with prominent myophagocytosis (arrow, A) and regeneration. Note presence of eosinophils in the infiltrate. In addition, neurogenic features with atrophic fibres with clumped myonuclei (arrow, B) and adipose tissue infiltration is also noted. (a: $\mathrm{H}$ and $\mathrm{E}, \times 120, \mathrm{~b}$ : $\mathrm{H}$ and $\mathrm{E}, \times 40$ )

\section{Discussion}

True hypertrophy is an increase in size involving all the different tissues composing the part whereas pseudohypertrophy is an abnormal enlargement of a structure caused by an overgrowth of fatty and fibrous tissue. In the present case, the enlarged muscle is pseudohypertrophied as it is weak and imaging and biopsy are consistent with fatty infiltration of the muscles. In disorders involving the motor neurons and their axons, muscle hypertrophy is uncommon. Focal muscle hypertrophy occurs most commonly in patients with lumbosacral radiculopathies ${ }^{[2]}$ and, less often, neuropathies or old polio. ${ }^{[3]}$ Disorders such as GuillainBarre syndrome,$^{[4]}$ chronic inflammatory demyelinating polyneuropathy (CIDP),$^{[5]}$ polio, ${ }^{[3]}$ amyloidosis, ${ }^{[6]}$ hypothyroidism, ${ }^{[7]}$ spinal muscular atrophy (SMA) and HMSN types 1 and 2, traumatic, ${ }^{[8]}$ or compressive nerve lesion with herniated disk or spinal stenosis ${ }^{[9]}$ have been associated with unilateral or bilateral calf hypertrophy. Muscle hypertrophy can occur both with abnormal motor activity such as myokymia, neuromyotonia, or continuous repetitive discharges (CRDs), as well as in instances without abnormal spontaneous motor activity. ${ }^{[1]}$

Muscle biopsy findings in enlarged muscles with myokymic discharges have revealed hypertrophy of type 1 myofibers in isolation, or of both fiber types. ${ }^{[6]}$ Fiber splitting is an additional finding as is fiber type grouping. ${ }^{[4]}$ In this subgroup, magnetic resonance image (MRI) of the enlarged gastrocnemius performed in a case revealed enlargement of gastrocnemius without an increase in adipose tissue content consistent with a true muscle hypertrophy. ${ }^{[5]}$ In patients with muscle hypertrophy associated with CRDs, the muscle biopsy reveals a variety of findings with neurogenic changes such as angulated atrophic fibers and target fibers, fibertype grouping, fiber splitting, and enlargement of both myofiber types. Other findings described include an increased number of internal nuclei, rimmed vacuoles, degenerating and regenerating myofibers, and increase in connective tissue reflecting a chronic neurogenic process. ${ }^{[1]}$ The chronic stimulation of the myofibers by CRDs might result in myofiber hypertrophy and fiber splitting seen on muscle biopsy, and increased muscle bulk seen on MRI and CT scan of some of the patients. ${ }^{[5,10]}$

In focal hypertrophy without spontaneous motor activity, biopsy of enlarged calf muscle might reveal only occasional atrophic fibers with a tendency to fiber-type grouping, while others show most muscle fibers to be hypertrophic with myofiber-type grouping and rare atrophic fibers. There was increased fat in the interstitial space and replacing muscle tissue. ${ }^{[10]} \mathrm{CT}$ scan findings of the enlarged muscle revealed true muscle hypertrophy in few cases with radiculopathy and areas of hypodensity consistent with increased connective and fat tissue in others. ${ }^{[10]}$ In biopsies of bilateral hypertrophy without spontaneous motor activity, hypertrophy is hypothesized to be due to a combination of genuine work hypertrophy of nondenervated muscle fibers and widespread endomysial fibrosis and fatty replacement. CT scan in such cases have shown muscle hypertrophy in gastrocnemius muscles in some patients with HMSN type 1 and the enlarged gastrocnemius diffusely infiltrated by fat in patients with HMSN type $2 .{ }^{[1]}$

The present case is interesting in that the whole of right lower limb is enlarged due to a neurogenic cause. Even though neurogenic hyper/pseudohypertrophy is well described in the literature ${ }^{[2-5,8,9]}$ all these reports mention isolated calf hypertrophy. To the best of our 
knowledge, even though whole of unilateral upper limb pseudohypertrophy in a patient with syringomyelia has been reported ${ }^{[11]}$ there are no reports of whole of lower limb being enlarged due to a neurogenic cause.

\section{References}

1. Gutmann L. AAEM Minimonograph \#46: Neurogenic muscle hypertrophy. Muscle Nerve 1996;19:811-8.

2. Bertorini T, Woodhouse C, Horner L. Muscle hypertrophy secondary to the tethered cord syndrome. Muscle Nerve 1994;17:331-5.

3. Donato G, Valentino P, Santucci M, Amorosi A, Pittelli M, Maltese L, et al. Calf pseudohypertrophy in a patient with double neurogenic pathology. Clin Neuropathol 2000;19:142-4.

4. Vasilescu C, Alexianu M, Dan A. Muscle hypertrophy and a syndrome of continuous motor unit activity in prednisone responsive Guillain-Barré polynewopathy. J Neurol 1984;231:276-9.

5. Pareyson D, Morandi I, Scaioli V, Marazzi R, Boiardi A, Sghirlanzoni A.
Neurogenic muscle hypertrophy. J Neurol 1989;236:292-5.

6. Gutmann LA, Gutmann LU, Schochet SS. Spontaneous repetitive discharges and muscle hypertrophy. Muscle Nerve 1993;16:1092.

7. Kaminski HJ, Ruff RL. Endocrine myopdthies, In: Engel AG, FranziniArmstrong C, editors. Myology. New York: McGraw-Hill; 1994;2: 1726-53.

8. Cirafisi C, Tezzon F, Curró Dossi B. Calf hypertrophy due to a iatrogenic sciatic nerve lesion: Case report. Ital J Neurol Sci 1992;13:251-4

9. Ong V, Jones J, Steuer A. An unusual cause of calf hypertrophy: Severe lumbar canal stenosis with S1 nerve root radiculopathy. APLAR J Rheumatol 2007;10:316-9.

10. Ricker K, Rohkamm R, Moxley RT. Hypertrophy of the calf with S1 radiculopathy. Arch Neurol 1988;45:660-4.

11. Mehta J, Khanna S. Syringomyelia as a cause of limb hypertrophy. Neurol India 2002;50:93-4.

How to cite this article: Netto $A B$, Sinha $S$, Taly $A B$, Prasad $C$, Mahadevan A, Bindu PS, et al. An unusual case of unilateral limb hypertrophy: Lipoma of sacral roots. J Neurosci Rural Pract 2012;3:89-92.

Source of Support: Nil. Conflict of Interest: None declared. 